\title{
On network stability, graph separation, interconnection structure and convex shells
}

\author{
Ioannis Lestas \\ Department of Engineering \\ University of Cambridge \\ Cambridge CB2 1PZ, UK \\ icl20@cam.ac.uk
}

\begin{abstract}
We consider a large scale network comprised of heterogeneous dynamical components. We derive conditions on the input/output properties of locally defined subsystems within the network, such that if these are satisfied the entire interconnection is guaranteed to be stable. The conditions are based on appropriately constructed functionals that lead to graph separation. We illustrate how interconnection structure can be exploited when determining the form of these functionals so as to reduce conservatism in the certificates. Interpretations of the stability conditions by means of disjoint convex shells are also discussed.
\end{abstract}

Index Terms - control of networks, complex systems, decentralized control, large scale systems.

\section{INTRODUCTION}

One of the main challenges in a large scale network is the ability to guarantee stability of the entire interconnection by conditions on only local subsystems. This is a problem that has received considerable attention by the control community from an early stage (e.g. the dissipativity approaches in [1], [2]), with a renewed interest in recent years due to the significance in many important applications such as data network protocols, group coordination problems and power distribution networks [3], [4], [5], [6], [7], [8], [9], [10]. A major complication in such a setting arises when the participating dynamics are heterogeneous and interconnection structure needs to be exploited in this case in order to avoid more conservative results that are based on weak interactions.

Our approach in the paper is to derive such decentralized conditions by means of functionals that lead to graph separation in $\mathscr{L}_{2}$, with stability following by means of appropriate homotopy arguments as in [11]. By exploiting interconnection structure we show how different classes of functionals can be considered that can reduce conservatism while maintaining decentralization. Furthermore it is shown how graph separation in this context can be interpreted by means of disjoint convex shells.

The paper builds upon our work in [12], and also other related works discussed throughout the paper, by incorporating richer classes of separating functionals, the form of which is determined by the interconnection structure. This allows to reduce conservatism while also retaining nonlinear interpretations.

The paper is structured as follows. The notation that will be used throughout the paper is first introduced. An overview on the connection between quadratic graph separation and stability is then provided, and various properties of the DavisWielandt shell are also mentioned. The main results are given in section III. Conditions for graph separation are derived first and it is then shown how these can lead to corresponding stability certificates. Conclusions are finally drawn in section IV. The proofs of the theorems in the paper have been omitted due to page constraints (these are included in an extended version of the paper [13]).

\section{Preliminaries}

\section{A. Notation}

Real/complex numbers are denoted by $\mathbb{R} / \mathbb{C}$ respectively and $\overline{\mathbb{R}}$ denotes the extended real line $[-\infty,+\infty]$. Nonnegative reals are denoted by $\mathbb{R}_{+}$, the halfplane $\{x \in \mathbb{C}: \Re(x) \geq \sigma\}$ is denoted by $\mathbb{C}_{\sigma^{+}}$, and $\overline{\mathbb{R}}_{+}:=\mathbb{R}_{+} \cup\{\infty\}$. For $x \in \mathbb{C}^{n}$, its Euclidean norm is denoted by $\|x\|$ and $\|x\|_{p}$ denotes its $\mathrm{p}$ norm. For a matrix $M \in \mathbb{C}^{n \times m}$ its conjugate transpose is denoted by $M^{*}$, its Moore-Penrose pseudoinverse by $M^{+}$, and $I_{n} \in \mathbb{R}^{n \times n}$ denotes the $n \times n$ identity matrix. For $M \in \mathbb{C}^{n \times n}$ $\operatorname{eig}(M)$ denotes its spectrum.

$\mathscr{L}_{2}^{l}[0, \infty)$ is the Hilbert space of functions $f:[0, \infty) \rightarrow$ $\mathbb{R}^{l}$ with finite norm $\|f\|=\langle f, f\rangle^{1 / 2}$ where $\langle f, g\rangle=$ $\int_{0}^{\infty} f^{*}(t) g(t) d t$. This is a subspace of $\mathscr{L}_{2 e}^{l}[0, \infty)$ whose elements need to be square integrable on finite intervals. The fourier transform of $f \in \mathscr{L}_{2}^{l}[0, \infty)$ is denoted by $\hat{f}(j \omega)=$ $\int_{0}^{\infty} e^{-j \omega t} f(t) d t$.

For some $T \geq 0, P_{T}: \mathscr{L}_{2 e}^{l}[0, \infty) \rightarrow \mathscr{L}_{2 e}^{l}[0, \infty)$ is the projection operator defined as $\left(P_{T}(f)\right)(t)=f(t)$ for $t \leq T$ and $\left(P_{T}(f)\right)(t)=0$ otherwise. An operator $\Delta: \mathscr{L}_{2 e}^{l}[0, \infty) \rightarrow$ $\mathscr{L}_{2 e}^{m}[0, \infty)$ is said to be causal if $P_{T} \Delta=P_{T} \Delta P_{T}$ for $T \geq 0$. This is bounded if the gain $\|\Delta\|=\sup \{\|\Delta(f)\| /\|f\|: f \in$ $\left.\mathscr{L}_{2}^{l}[0, \infty), f \neq 0\right\}$ exists and is finite.

The Kronecker product is denoted by $\otimes$, and for $\Pi: \mathbb{C} \rightarrow$ $\mathbb{C}^{n \times m}, A \in \mathbb{C}^{p \times q}$ we denote $\Pi \otimes A$ the function $\Pi \otimes A: \mathbb{C} \rightarrow$ $\mathbb{C}^{n p \times m q},(\Pi \otimes A)(s)=\Pi(s) \otimes A$. The direct sum of operators $\Delta_{i} i=1, \ldots n$ is denoted by $\oplus_{i=1}^{n} \Delta_{i}$. For a set $A$ in a real vector space, $\operatorname{Co}(A)$ denotes its convex hull.

We say that convex sets $A, B \subset \mathbb{R}^{n}$ are $\varepsilon$-strictly separated for some $\varepsilon>0$ if there exists a $v \in \mathbb{R}^{n}$ and $a \in \mathbb{R}$ such that $x^{*} v \geq \varepsilon+a \forall v \in A$ and $x^{*} v \leq a \forall x \in B$. We also say in this case that the hyperplane $\left\{x: x \in \mathbb{R}^{n}, x^{*} v=a\right\}$ strictly separates $A$ and $B$. It is known from the separating hyperplane theorem that if $A, B \subset \mathbb{R}^{n}$ are non-empty, closed, convex sets 
with at least one compact, then $A, B$ being disjoint implies that they are strictly separated by a hyperplane (e.g. [14]).

A directed graph is denoted by $\mathscr{G}=(V, E)$, where $V=$ $\left\{v_{1}, \ldots, v_{n}\right\}$ is the set of nodes and $E \subseteq V \times V$, the set of directed edges. The adjacency matrix $A \in \mathbb{R}^{n \times n}$ of the graph is defined as $A_{i j}=1 \Leftrightarrow\left(v_{i}, v_{j}\right) \in E$ and $A_{i j}=0$ otherwise. A directed cycle is an ordered set of vertices $\left\{v_{0}, v_{1}, \ldots, v_{N}, v_{0}\right\}$ such that $v_{0}, \ldots, v_{N}$ are distinct with $\left(v_{i-1}, v_{i}\right) \in E \forall i \in$ $\{1,2, \ldots, N\}$ and also $\left(v_{N}, v_{0}\right) \in E$. We define a directed graph as circular if it has a single directed cycle. A directed graph is defined to be bipartite if its set of vertices can be decomposed as $V=A \cup B$, where $A$ and $B$ are two disjoint sets with any edge $e_{k}=\left(v_{i}, v_{j}\right)$ satisfying $v_{i} \in A$ and $v_{j} \in B$, or $v_{j} \in A$ and $v_{i} \in B$. For a matrix $M \in \mathbb{C}^{n \times n}$ we define its underlying graph as the directed graph with adjacency matrix $A \in \mathbb{R}^{n \times n}$ such that $A_{i j}=0$ iff $M_{i j}=0$ and $A_{i j}=1$ otherwise.

We will be using the algebra of transfer functions introduced in [15], [16], [17] (this has properties analogous to those of proper real rational transfer functions). $\mathscr{A}\left(\sigma_{0}\right)$ denotes the class of transfer functions obtained as the Laplace transform of impulse response functions $h=h_{a}+\sum_{i=0}^{\infty} k_{i} \delta_{t_{i}}$ where $h_{a}: \mathbb{R} \rightarrow \mathbb{C}$ satisfies $\int_{0}^{\infty} e^{-\sigma_{0} t}|h(t)| d t<\infty$ and $h_{a}(t)=0$ for $t \leq 0, t_{0}=0, t_{i}>0$ for $i>0, k_{i} \in \mathbb{C}$ s.t. $\sum_{i=0}^{\infty} e^{-\sigma_{0} t_{i}}\left|k_{i}\right|<\infty$ and $\delta_{t_{i}}$ denotes the $t_{i}$ shifted Dirac delta distribution. Functions $g \in \mathscr{A}\left(\sigma_{0}\right)$ are bounded on $\mathbb{C}_{\sigma_{0}^{+}}$, and $\omega \rightarrow g(\sigma+j \omega)$ is continuous on $\overline{\mathbb{R}}$ for $\sigma \geq \sigma_{0}$. Any $g \in \mathscr{A}\left(\sigma_{0}\right)$ defines in the time domain a bounded causal operator in $\mathscr{L}_{2 e}[0, \infty)$ defined by a convolution integral [15]-[17]. We will denote by $\mathscr{A}$ the transfer functions that are in $\mathscr{A}\left(\sigma_{0}\right)$ for some $\sigma_{0}<0$ and also these are constant at infinity in $\mathbb{C}_{\sigma_{0}^{+}} \cdot \mathscr{A}^{n \times m}$ denotes the $n \times m$ transfer matrices with elements in $\mathscr{A}$. We also denote $\mathscr{A}^{n \times n}$ the transfer matrices $A \in \mathscr{A}^{n \times n}$ such that for almost all $\omega \in \mathbb{R}, A(j \omega)$ and $A^{*}(j \omega)$ have the same nullspace (this is for example the case when $A(j \omega)$ is invertible or normal).

\section{B. Quadratic graph separation and stability}

Consider the interconnection of operators $G: \mathscr{L}_{2 e}^{l}[0, \infty) \rightarrow$ $\mathscr{L}_{2 e}^{m}[0, \infty)$ and $\Delta: \mathscr{L}_{2 e}^{m}[0, \infty) \rightarrow \mathscr{L}_{2 e}^{l}[0, \infty)$

$$
\begin{gathered}
v=G(w)+f \\
w=\Delta(v)+e
\end{gathered}
$$

where $f \in \mathscr{L}_{2 e}^{l}[0, \infty)$ and $e \in \mathscr{L}_{2 e}^{m}[0, \infty)$.

Definition 1 (well-posedness, stability): We say that the feedback interconnection of operators $G$ and $\Delta$ is well-posed if the map $(v, w) \rightarrow(e, f)$ in (1) has an inverse that is a causal operator on $\mathscr{L}_{2 e}^{l+m}[0, \infty)$. The interconnection is stable if this operator is also bounded.

We will be considering in this section an IQC framework for verifying stability of the interconnection [11], [18]. This requires that the graphs of the interconnected operators be separated in $\mathscr{L}_{2}^{l+m}[0, \infty)$, with stability guaranteed by means of an additional homotopy argument that ensures there is no transition from stability to instability.

For an operator $\Delta: \mathscr{L}_{2 e}^{m}[0, \infty) \rightarrow \mathscr{L}_{2 e}^{l}[0, \infty)$ its graph is defined as

$$
\mathscr{G}_{\Delta}:=\left\{(\Delta(v), v): v \in \mathscr{L}_{2}^{l}[0, \infty), \Delta(v) \in \mathscr{L}_{2}^{m}[0, \infty)\right\}
$$

and its inverse graph as

$$
\overline{\mathscr{G}}_{\Delta}:=\left\{(v, \Delta(v)): v \in \mathscr{L}_{2}^{l}[0, \infty), \Delta(v) \in \mathscr{L}_{2}^{m}[0, \infty)\right\}
$$

Operator $\Delta_{\tau}: \mathscr{L}_{2 e}^{m}[0, \infty) \rightarrow \mathscr{L}_{2 e}^{l}[0, \infty)$ is said to depend continuously on $\tau$ if $\delta\left(\Delta_{\tau_{1}}, \Delta_{\tau_{2}}\right) \rightarrow 0$ as $\left|\tau_{2}-\tau_{1}\right| \rightarrow 0$, where $\delta\left(\Delta_{\tau_{1}}, \Delta_{\tau_{2}}\right)$ is as defined in [18],

$$
\begin{aligned}
& \vec{\delta}\left(\Delta_{\tau_{1}}, \Delta_{\tau_{2}}\right):=\sup _{g \in S_{\Delta \tau_{1}}} \inf _{h \in S_{\Delta \tau_{2}} T>0,\left\|P_{T} g\right\| \neq 0} \sup _{\| P_{T}} \frac{\left\|P_{T} g-P_{T} h\right\|}{\left\|P_{T} g\right\|} \\
& \delta\left(\Delta_{\tau_{1}}, \Delta_{\tau_{2}}\right):=\max \left(\vec{\delta}\left(\Delta_{\tau_{1}}, \Delta_{\tau_{2}}\right), \vec{\delta}\left(\Delta_{\tau_{2}}, \Delta_{\tau_{1}}\right)\right)
\end{aligned}
$$

with $S_{\Delta_{\tau_{i}}}:=\left\{\left(\Delta_{\tau_{i}}(v), v\right): v \in \mathscr{L}_{2 e}^{m}[0, \infty)\right\}$ denoting the extended graph of operator $\Delta_{\tau_{i}}$ (distance $\delta$ is close to the definition of the gap ' $\delta_{0}$ ' in [19]).

For example, $\Delta_{\tau}=\tau \Delta$ depends continuously on $\tau$ when operator $\Delta$ is bounded. This is the case since

$$
\begin{aligned}
\frac{\left\|P_{T}\left(\Delta_{\tau_{1}}(w), w\right)-P_{T}\left(\Delta_{\tau_{2}}(w), w\right)\right\|}{\left\|P_{T}\left(\Delta_{\tau_{1}}, w\right)\right\|} & \leq \frac{\left|\tau_{1}-\tau_{2}\right|\left\|P_{T} \Delta(w)\right\|}{\left\|P_{T}(w)\right\|} \\
& \leq\left|\tau_{1}-\tau_{2}\right|\|\Delta\|
\end{aligned}
$$

A functional $\sigma: \mathscr{L}_{2}^{n}[0, \infty) \rightarrow \mathbb{R}$ is said to be quadratically continuous [18] if for every $\varepsilon>0$ there exists $C>0$ such that

$$
\sigma(h) \leq \sigma(g)+\varepsilon\|g\|^{2}+C\|h-g\|^{2}, \quad \forall g, h \in \mathscr{L}_{2}^{n}[0, \infty)
$$

We will be using quadratically continuous functionals of the form

$$
\sigma_{\Pi}(h):=\int_{-\infty}^{\infty} \hat{h}(j \omega)^{*} \Pi(j \omega) \hat{h}(j \omega) d \omega
$$

where $\Pi: j \mathbb{R} \rightarrow \mathbb{C}^{n}$ is a Hermitian valued function. Nonnegativity of $\sigma_{\Pi}(h)$ for all $h \in \mathscr{L}_{2}^{n}[0, \infty)$ can be checked in the frequency domain as stated in the following Lemma (e.g. [20]).

Lemma 1: Let $\sigma_{\Pi}: \mathscr{L}_{2}^{n}[0, \infty) \rightarrow \mathbb{R}$ be defined as in (2). Then $\sigma_{\Pi}(h) \geq 0 \forall h \in \mathscr{L}_{2}^{n}[0, \infty)$ iff $\Pi(j \omega) \geq 0$ for almost all $\omega \in \mathbb{R}_{+}$.

Definition 2 (quadratic graph separation): We say that the graphs of operators $G: \mathscr{L}_{2 e}^{l}[0, \infty) \rightarrow \mathscr{L}_{2 e}^{m}[0, \infty)$ and $\Delta$ : $\mathscr{L}_{2 e}^{m}[0, \infty) \rightarrow \mathscr{L}_{2 e}^{l}[0, \infty)$ are $\varepsilon$-quadratically separated by the functional $\sigma: \mathscr{L}_{2}^{l+m}[0, \infty) \rightarrow \mathbb{R}$ if $\sigma$ is quadratically continuous, $\varepsilon>0$ and

$$
\begin{array}{lll}
\sigma(g) \geq \varepsilon\|g\|^{2} & \forall g \in \mathscr{G}_{G} \\
\sigma(h) \leq 0 & \forall h \in \overline{\mathscr{G}}_{\Delta}
\end{array}
$$

Quadratic separation of the graphs of the operators is not sufficient for the interconnection in (1) to be stable, but it ensures that stability is preserved if these are perturbed continuously from a stable interconnection. This is stated in the Theorem below from [18].

Theorem 1: Suppose that the interconnection of operators $G_{\tau}: \mathscr{L}_{2 e}^{l}[0, \infty) \rightarrow \mathscr{L}_{2 e}^{m}[0, \infty)$ and $\Delta_{\tau}: \mathscr{L}_{2 e}^{m}[0, \infty) \rightarrow \mathscr{L}_{2 e}^{l}[0, \infty)$ is

(i) stable for $\tau=0$, well posed for $\tau \in[0,1]$, and $G_{\tau}, \Delta_{\tau}$ depend continuously on $\tau$,

(ii) there exists $\varepsilon>0$ and a quadratically continuous functional $\sigma: \mathscr{L}_{2}^{l+m}[0, \infty) \rightarrow \mathbb{R}$ s.t. for all $\tau \in[0,1]$ the graphs of $G_{\tau}$ and $\Delta_{\tau}$ are $\varepsilon$-quadratically separated by $\sigma$, then the interconnection of $G_{1}$ and $\Delta_{1}$ is stable. 


\section{The Davis-Wielandt shell and convexity of joint ranges}

Let $B(\mathscr{H})$ be the algebra of bounded linear operators acting on the Hilbert space $\mathscr{H}$ with inner product $\langle g, h\rangle$ for $g, h \in \mathscr{H}$. The numerical range of $A \in B(\mathscr{H})$ is defined by

$$
W(A)=\{\langle A x, x\rangle: x \in \mathscr{H},\langle x, x\rangle=1\}
$$

The Davis-Wielandt shell [21], [22] of $A \in B(\mathscr{H})$ is a higher dimensional generalization of the numerical range which is defined by

$D W(A)=\{(\Re\langle A x, x\rangle, \mathfrak{I}\langle A x, x\rangle,\langle A x, A x\rangle): x \in \mathscr{H},\langle x, x\rangle=1\}$

The following properties follow from its definition and are analogous to ones satisfied by the numerical range (e.g. [23])

Lemma 2: Let $A \in B(\mathscr{H})$. Then

(a) $D W(A)$ is bounded.

(b) $D W(A)=D W\left(U^{*} A U\right)$ for any unitary $U \in B(\mathscr{H})$.

(c) Suppose $A$ is the direct sum $A_{1} \oplus \ldots \oplus A_{n}$. Then

$$
D W(A)=C o\left\{D W\left(A_{1}\right) \cup \ldots \cup D W\left(A_{n}\right)\right\}
$$

(d) $D W(A)$ is closed if $\operatorname{dim}(\mathscr{H})$ is finite.

The Davis-Wielandt shell captures more information about the operator relative to the numerical range as indicated in the Lemma below (e.g. [23], [21]).

Lemma 3: Let $A \in B(\mathscr{H})$ with spectrum $\sigma(A)$, and $\operatorname{dim}(\mathscr{H})$ finite. Then

$$
D W(A)=\operatorname{Co}\left\{\left(\Re(\lambda), \mathfrak{I}(\lambda),|\lambda|^{2}\right): \lambda \in \sigma(A)\right\}
$$

iff $A$ is normal.

It should be noted that if the sets in the Lemma are projected to their first two coordinates (i.e. the numerical range was used instead of the Davis-Wielandt shell), the 'only if' part would not be true.

It is known from the Toeplitz-Hausdorff theorem that the numerical range of $A \in B(\mathscr{H})$ is convex. This is, however, not always the case for the Davis-Wielandt shell. The convexity properties of $D W(A)$ depend on $n=\operatorname{dim}(\mathscr{H})$ and can be deduced from corresponding results on the joint range of $m$-tuples of Hermitian forms.

A Hermitian form $\phi$ on a normed vector space $V$ over $\mathbb{C}$ is defined as a functional $\phi(x)=f(x, x)$ where $f: V \times V \rightarrow \mathbb{C}$, $f(x, y)$ is linear in $x$ and $[f(x, y)]^{*}=f(y, x)$. Note that $D W(A)$ can be written as the 3-tuple of Hermitian forms

$$
\begin{array}{r}
\left\{\left\langle x, \frac{1}{2}\left(A+A^{*}\right) x\right\rangle,-j\left\langle x, \frac{1}{2}\left(A-A^{*}\right) x\right\rangle,\langle A x, A x\rangle\right. \\
: x \in \mathscr{H},\langle x, x\rangle=1\}
\end{array}
$$

where $A^{*}$ denotes the adjoint of $A$. It follows from the Theorem below that $D W(A)$ is either a convex set or an ellipsoid.

Theorem 2: [24] Let $V$ be a vector space over $\mathbb{C}$ with $U=\{x \in V:\|x\|=1\}$ and let $\phi=\left(\phi_{1}, \ldots, \phi_{m}\right)$ be an $m$-tuple of Hermitian forms on $V$.

(i) If $\operatorname{dim}(V)=1$ then $\phi(U)$ is a point.

(ii) If $\operatorname{dim}(V)=2$ then $\phi(U)$ is an affine image of the unit sphere $\left\{x \in \mathbb{R}^{3}:\|x\|=1\right\}$. (iii) If $\operatorname{dim}(V)>2$ and $m \leq 3$ then $\phi(U)$ is convex.

The following corollary follows directly from Theorem 2 and Lemma 3.

Corollary 1: Let $A \in B(\mathscr{H})$. Then $D W(A)$ is convex if $\operatorname{dim}(\mathscr{H}) \neq 2$ or $A$ is normal, and an ellipsoid if $\operatorname{dim}(\mathscr{H})=2$.

The Davis-Wielandt shell is therefore either a convex set or encloses a convex set. It can hence be characterized by means of computationally efficient techniques analogous to the ones used for the numerical range [23]. That is, points/support hyperplanes can easily be generated on its boundary, and these can then be used to derive lower/upper bounds for the shell with arbitrarily high precision.

For a matrix $A \in \mathbb{C}^{n \times n}$ with Moore-Penrose pseudoinverse $A^{+}$we denote the shell of $A^{+}$restricted to $\operatorname{range}(A)$ as

$$
\begin{array}{r}
\tilde{D W}\left(A^{+}\right):=\left\{\left(\mathfrak{R}\left(x^{*} A^{+} x\right), \mathfrak{I}\left(x^{*} A^{+} x\right),\left\|A^{+} x\right\|^{2}\right)\right. \\
\left.: x \in \mathbb{C}^{n},\|x\|=1, x \in \operatorname{range}(A)\right\}
\end{array}
$$

and define

$$
\overline{D W}(A):= \begin{cases}D \tilde{W}\left(A^{+}\right) & \text {if } \operatorname{rank}(A)=n \\ D \tilde{W}\left(A^{+}\right)+\left\{0,0, \mathbb{R}_{+}\right\} & \text {if } \operatorname{rank}(A)<n\end{cases}
$$

i.e. $\overline{D W}(A)=D W\left(A^{-1}\right)$ if $A$ is invertible. If $A$ is normal then $\tilde{D W}\left(A^{+}\right)$simplifies in an analogous way to $D W(A)$ as shown in the Lemma below from [12].

Lemma 4: Let $A \in \mathbb{C}^{n \times n}$ be normal. Then

$$
D \tilde{W}\left(A^{+}\right)=\operatorname{Co}\left\{\left(\Re \frac{1}{\lambda}, \mathfrak{I} \frac{1}{\lambda}, \frac{1}{|\lambda|^{2}}\right): \lambda \neq 0, \lambda \in \sigma(A)\right\} \text {. }
$$

\section{MAIN RESULTS}

We consider an interconnection as in (1)

$$
\begin{aligned}
v & =G(w)+f \\
w & =A(v)+e
\end{aligned}
$$

where $G=\oplus_{i=1}^{n} G_{i}$ the direct sum of operators $G_{i}$ : $\mathscr{L}_{2 e}^{n_{i}}[0, \infty) \rightarrow \mathscr{L}_{2 e}^{n_{i}}[0, \infty)$ and operator $A: \mathscr{L}_{2 e}^{\sum_{i} n_{i}}[0, \infty) \rightarrow$ $\mathscr{L}_{2 e}^{\sum_{i} n_{i}}[0, \infty)$ has compatible dimensions.

This can be seen as a network comprised of subsystems $G_{i}$, and with the interconnections between them determined by means of the operator $A$. The aim is to derive scalable stability conditions for the network that are based on conditions on the input-output properties of the individual subsystems $G_{i}$, and an exploitation of structural properties of the interconnection operator $A$. Such properties will be discussed throughout this section with examples given from circular and bipartite graphs, an important class of graphs that is relevant in many applications such as group coordination problems and Internet congestion control. Stability will be verified through conditions that ensure quadratic separation of the graphs of operator $\oplus G_{i}$ and $A$. These separating functionals are convenient in this context for the following reasons:

(i) They have some convenient decomposition properties that can lead to decentralized conditions, with the form of the functionals determined from known structural properties of the interconnection operator. 
(ii) Easily verifiable conditions can be obtained for certain classes of functionals (such as conditions involving disjoint convex shells that will be discussed below), which could also motivate the choice of the separating functionals for given classes of dynamics the network is comprised of.

(iii) Stability of interconnections of nonlinear operators can also be deduced if these satisfy the input/output properties defined by the separating functionals.

In order to better illustrate the ideas that lead to decentralized conditions we will consider first the problem of establishing quadratic graph separation, as this is the key step where interconnection structure is exploited. Stability can then be deduced by means of appropriate homotopy arguments as in Theorem 1.

\section{A. Graph separation}

The Theorem below follows directly from the definition of the functionals in $^{1}$ (2). To state the Theorem we define for $i=1, \ldots, n$ functionals

$$
\sigma_{\Pi^{i}}: \mathscr{L}_{2}^{2 n_{i}}[0, \infty) \rightarrow \mathbb{R}, \quad \sigma_{\tilde{\Pi}}: \mathscr{L}_{2}^{2 \sum_{i} n_{i}}[0, \infty) \rightarrow \mathbb{R}
$$

as in (2) where

$$
\Pi^{i}=\left[\begin{array}{ll}
\Pi_{11}^{i} & \Pi_{12}^{i} \\
\Pi_{12}^{i *} & \Pi_{22}^{i}
\end{array}\right], \quad \tilde{\Pi}:=\left[\begin{array}{ll}
\oplus_{i} \Pi_{11}^{i} & \oplus_{i} \Pi_{12}^{i} \\
\oplus_{i} \Pi_{12}^{i *} & \oplus_{i} \Pi_{22}^{i}
\end{array}\right]
$$

with $\Pi_{11}^{i}, \Pi_{12}^{i}, \Pi_{22}^{i}$ taking values in $\mathbb{C}^{n_{i} \times n_{i}}$.

Theorem 3: The following are equivalent for the operators $G_{i}$ and $A$ in (3) and the functionals $\sigma_{\Pi^{i}}, \sigma_{\tilde{\Pi}}$ in (4):

(i)

$$
\begin{array}{lll}
\sigma_{\Pi^{i}}(h) \geq \varepsilon\|h\|^{2} & \forall h \in \mathscr{G}_{G_{i}} \text { and } \forall i=1, \ldots, n & \text { and } \\
\sigma_{\tilde{\Pi}}(k) \leq 0 & & \leq k \in \overline{\mathscr{G}}_{A} .
\end{array}
$$

(ii) The graphs of $\oplus_{i=1}^{n} G_{i}$ and $A$ are $\varepsilon$-quadratically separated by $\sigma_{\tilde{\Pi}}$.

The key feature in Theorem 3 is that choosing the multipliers to have the same sparsity structure as that of $\oplus G_{i}$ then these decompose into a set of decentralized conditions. The corresponding conditions on the interconnection operator can be seen as constraints that are imposed on the multipliers. These can lead to some generic easily verifiable restrictions under further structural assumptions on the interconnection matrix and the allowable multipliers.

For example consider the following case considered in [25] where we have the interconnection in (3) with $n_{i}=m$, for $i=1, \ldots n$, interconnection operator $A$ is linear with transfer function

$$
\hat{A}=\hat{\Gamma} \otimes I_{m}, \quad \hat{\Gamma} \in \mathscr{A}^{n \times n}
$$

and $\hat{\Gamma}(j \omega)$ is normal for all $\omega \in \mathbb{R}$. The following Theorem from [25] then holds.

\footnotetext{
${ }^{1}$ It should be noted that analogous decompositions have been used from an early stage within the context of dissipative systems [1], where real valued multipliers were used to establish graph separation in $\mathscr{L}_{2 e}$.
}

Theorem 4: The graphs of operators $\oplus G_{i}$ and $A$ in (3), (6) are $\varepsilon$-quadratically separated, if there exists $\varepsilon>0$ and $\sigma_{\Pi}$ as defined in (2) with

$$
\Pi=\left[\begin{array}{ll}
\Pi_{11} & \Pi_{12} \\
\Pi_{12}^{*} & \Pi_{22}
\end{array}\right]
$$

and $\Pi_{11}, \Pi_{12}, \Pi_{22}$ taking values in $\mathbb{C}^{m \times m}$, such that

(i) $\sigma_{\Pi}(h) \geq \varepsilon\|h\|^{2} \quad \forall h_{i} \in \mathscr{G}_{G_{i}} \quad i=1, \ldots, n$

(ii) $\left(|\lambda|^{2} \Pi_{11}+\lambda^{*} \Pi_{12}+\lambda \Pi_{12}^{*}+\Pi_{22}\right)(j \omega) \leq 0 \quad \forall \lambda \in$ $\operatorname{eig}(\hat{\Gamma}(j \omega)), \forall \omega \in \mathbb{R}_{+}$

It should be noted that if there is more structure in the underlying graph of $\hat{\Gamma}$, such as that of a circular graph, then richer classes of multipliers can be considered without affecting scalability, i.e. the additional allowable parameters in the multipliers are independent of the interconnection matrix. This is shown in the Theorem below.

Theorem 5: Consider the interconnection in (3), (6) where the underlying graph of $\hat{\Gamma}$ is circular. The graphs of operators $\oplus G_{i}$ and $A$ are $\varepsilon$-quadratically separated, if $\exists \varepsilon>0$ and for $i=1, \ldots, n \exists \sigma_{\Pi^{i}}$ as defined in (2) with

$$
\Pi^{i}=\left[\begin{array}{ll}
M^{i^{*}} \Pi_{11} M^{i} & M^{i^{*}} \Pi_{12} M^{i} \\
M^{i^{*}} \Pi_{12}^{*} M^{i} & M^{i^{*}} \Pi_{22} M^{i}
\end{array}\right]
$$

where $M^{i}: j \mathbb{R} \rightarrow \mathbb{C}^{m \times m}, \Pi_{11}: j \mathbb{R} \rightarrow \mathbb{C}^{m \times m}, \Pi_{12}: j \mathbb{R} \rightarrow$ $\mathbb{C}^{m \times m}, \Pi_{22}: j \mathbb{R} \rightarrow \mathbb{C}^{m \times m}$ such that

(i) $\sigma_{\Pi^{i}}(h) \geq \varepsilon\|h\|^{2} \quad \forall h_{i} \in \mathscr{G}_{G_{i}} \quad i=1, \ldots, n$

(ii) $\left(|\lambda|^{2} \Pi_{11}+\lambda^{*} \Pi_{12}+\lambda \Pi_{12}^{*}+\Pi_{22}\right)(j \omega) \leq 0 \quad \forall \lambda \in$ $\operatorname{eig}(\hat{\Gamma}(j \omega)), \forall \omega \in \mathbb{R}_{+}$

If now the multipliers are scalar valued then graph separation can be deduced in a convenient way whereby DavisWieland shells are required to be disjoint pointwise over frequency. This follows from the Theorem below [12].

Theorem 6: The following are equivalent for operators $G_{i}$ with transfer functions $\hat{G}_{i} \in \mathscr{A}^{n_{i} \times n_{i}}$ for $i=1, \ldots, n$ and an operator $A$ with transfer function $\hat{A} \in \tilde{\mathscr{A}}^{N \times N}, N:=\sum_{i} n_{i}$ :

(i) $\exists$ functionals $\sigma_{\Pi \otimes I_{n_{i}}}: \mathscr{L}_{2}^{2 n_{i}}[0, \infty) \rightarrow \mathbb{R}$ as defined in (2) and $\varepsilon>0$ s.t.

$$
\begin{array}{lll}
\sigma_{\Pi \otimes I_{n_{i}}}(h) \geq \varepsilon\|h\|^{2} & \forall h \in \mathscr{G}_{G_{i}} \text { and } \forall i=1, \ldots, n \quad \text { and } \\
\sigma_{\Pi \otimes I_{N}}(k) \leq 0 & \forall k \in \overline{\mathscr{G}}_{A} . &
\end{array}
$$

(ii) $\exists$ functional $\sigma_{\Pi \otimes I_{N}}: \mathscr{L}_{2}^{2 N}[0, \infty) \rightarrow \mathbb{R}$ as defined in (2) and $\varepsilon>0$ s.t. the graphs of $\oplus_{i=1}^{n} G_{i}$ and $A$ are $\varepsilon$ quadratically separated by $\sigma_{\Pi \otimes I_{N}}$.

(iii) $\exists \varepsilon>0$ s.t. for almost all $\omega \in \mathbb{R}_{+}$

$$
\left.C o\left(\cup_{i} D W\left(\hat{G}_{i}(j \omega)\right)\right)\right) \text { and } \operatorname{Co}(\overline{D W}(\hat{A}(j \omega)))
$$

are $\varepsilon$-strictly separated.

(iv) $\exists \varepsilon>0$ s.t. for almost all $\omega \in \mathbb{R}_{+}$

$$
\left.\left.D W\left(\oplus_{i=1}^{n} \hat{G}_{i}(j \omega)\right)\right)\right) \text { and } C o(\overline{D W}(\hat{A}(j \omega)))
$$

are $\varepsilon$-strictly separated.

Remark 1: If $\hat{A}(j \omega)$ is normal then the disjoint shell conditions (iii), (iv) depend only on the spectrum of $\hat{A}(j \omega)$ (see Lemma 3, Lemma 4).

Note also that restricting the multipliers (and the corresponding separating functionals) to be of the form used in 
Theorem 6, allows to consider a full block interconnection matrix $A$, rather than require this to have the sparsity structure in (6).

Remark 2: It can be shown from the proof of the Theorem that the multipliers $\Pi(j \omega)$ define a frequency dependent separating hyperplane between the convex shells in (iii), (iv).

Remark 3: The conditions in Theorems 4-6 can lead to decentralized conditions for graph separation that hold for arbitrary networks in the following sense. If the spectrum of the interconnection matrix $A$ (or its Davis-Wielandt shell in Theorem 6) can be guaranteed to lie in a prescribed region (e.g. by appropriate scaling) then multipliers $\Pi$ can be chosen that always satisfy condition (ii) in Theorems 4 and 5; hence condition (i) becomes a decentralized condition on each of the individual subsystems. Similarly in Theorem 6 a separating hyperplane can be chosen such that this does not intersect the shell of $A$, and then each of the individual shells $D W\left(\hat{G}_{i}(j \omega)\right)$ should lie on the opposite side of this hyperplane.

We consider now an interconnection corresponding to a bipartite graph. That is we have

$$
\begin{aligned}
& y=K\left(\oplus_{i=1}^{n_{p}} P_{i}\right) u+e \\
& u=H\left(\oplus_{k=1}^{n_{q}} Q_{k}\right) y
\end{aligned}
$$

where

$$
\begin{gathered}
P_{i}: \mathscr{L}_{2 e}^{l_{p_{i}}}[0, \infty) \rightarrow \mathscr{L}_{2 e}^{m_{p_{i}}}[0, \infty), \quad i=1, \ldots, n_{p} \\
Q_{k}: \mathscr{L}_{2 e}^{l_{q_{k}}}[0, \infty) \rightarrow \mathscr{L}_{2 e}^{m_{q_{k}}}[0, \infty), \quad k=1, \ldots, n_{q}
\end{gathered}
$$

define the subsystems, and operators

$$
\begin{gathered}
H: \mathscr{L}_{2 e}^{\sum_{k} m_{q_{k}}}[0, \infty) \rightarrow \mathscr{L}_{2 e}^{\sum_{i} l_{p_{i}}}[0, \infty) \\
K: \mathscr{L}_{2 e}^{\sum_{i} m_{p_{i}}}[0, \infty) \rightarrow \mathscr{L}_{2 e}^{\sum_{k} l_{q_{k}}}[0, \infty)
\end{gathered}
$$

define the interconnections between them. A way this can be brought into the form (3) is by writing

$$
G=\left[\begin{array}{cc}
\oplus_{i=1}^{n_{p}} P_{i} & 0 \\
0 & \oplus_{k=1}^{n_{q}} Q_{k}
\end{array}\right], \quad A=\left[\begin{array}{cc}
0 & H \\
K & 0
\end{array}\right]
$$

The results presented in the previous paragraphs could then be used to deduce graph separation of $\oplus G_{i}$ and $A$. For example, if $l_{p_{i}}=m_{p_{i}}=l_{q_{k}}=m_{q_{k}}$ and the interconnection operator $A$ is linear and normal with a sparsity structure as in (6) then Theorem 4 can be used to derive corresponding decentralized conditions. In particular, if $\rho(\hat{A}(j \omega)) \leq 1$ and $\hat{A}(j \omega)$ is hermitian then we have $\operatorname{eig}(\hat{A}(j \omega)) \subset[-1,1]$ or $\operatorname{eig}(\hat{A}(j \omega)) \subset\{\alpha j:-1 \leq \alpha \leq 1\}$ if $\hat{A}(j \omega)$ is skew-hermitian.

Note also that $\overline{D W}(A(j \omega)$ has in this case also a nice characterization (this is analogous to the instability region in [8]), and the following corollary holds.

Corollary 2: Consider the interconnection in (3), (10) where $P_{i}, Q_{k}$ and $A$ are linear operators with transfer functions $\hat{P}_{i} \in \mathscr{A}^{m_{p_{i}} \times m_{p_{i}}}, \quad \hat{Q}_{k} \in \mathscr{A}^{m_{q_{k}} \times m_{q_{k}}}, \hat{A} \in$ $\mathscr{A}^{\left(\sum_{i} m_{p_{i}}+\sum_{k} m_{q_{k}}\right) \times\left(\sum_{i} m_{p_{i}}+\sum_{k} m_{q_{k}}\right)}$ and $\rho(\hat{A}(j \omega)) \leq 1$. The graphs of operators $G$ and $A$ are $\varepsilon$-quadratically separated if $\exists \varepsilon>0$ s.t. $\forall \omega \in \mathbb{R}_{+}$either of the following holds: (i) $\hat{A}(j \omega)$ is hermitian and

$$
C o\left(\cup_{i} D W\left(\hat{P}_{i}(j \omega)\right) \cup \cup_{k} D W\left(\hat{Q}_{k}(j \omega)\right)\right) \text { and } R_{1}
$$

are $\varepsilon$ strictly separated.

(ii) $\hat{A}(j \omega)$ is skew-hermitian and

$$
C o\left(\cup_{i} D W\left(\hat{P}_{i}(j \omega)\right) \cup \cup_{k} D W\left(\hat{Q}_{k}(j \omega)\right)\right) \text { and } R_{2}
$$

are $\varepsilon$ strictly separated

where sets $R_{1}$ and $R_{2}$ are given by

$$
\begin{aligned}
& R_{1}:=\operatorname{Co}\left\{1 / x, 0,1 / x^{2}:-1 \leq x \leq 1\right\} \\
& R_{2}:=\operatorname{Co}\left\{0,1 / x, 1 / x^{2}:-1 \leq x \leq 1\right\}
\end{aligned}
$$

We consider now an alternative way the interconnection in (7) can be brought to the form (3). In particular, using ideas from [5] we define the local subsystems $G_{i}$ and the interconnection operator $A$ as ${ }^{2}$

$$
G_{i}=K_{i}^{c} P_{i} H_{i}^{r}\left(\oplus_{k=1}^{n_{q}} Q_{k}\right), \quad A=M M^{*}
$$

where $P_{i}, Q_{k}$ are as in (8), (9) and

$$
\begin{aligned}
& H=\left[\begin{array}{c}
H_{1}^{r} \\
\vdots \\
H_{n_{p}}^{r}
\end{array}\right], \quad H_{i}^{r}=\left[\begin{array}{lll}
\tilde{H}_{i 1} & \ldots & \tilde{H}_{i n_{q}}
\end{array}\right] \quad \text { for } i=1, \ldots, n_{p} \\
& K=\left[\begin{array}{lll}
K_{1}^{c} & \ldots & K_{n_{p}}^{c}
\end{array}\right], \quad K_{i}^{c}=\left[\begin{array}{c}
\tilde{K}_{1 i} \\
\vdots \\
\tilde{K}_{n_{q} i}
\end{array}\right] \quad \text { for } i=1, \ldots, n_{q} \\
& \tilde{H}_{i k}: \mathscr{L}_{2 e}^{m_{q_{k}}}[0, \infty) \rightarrow \mathscr{L}_{2 e}^{l_{p_{i}}}[0, \infty), \tilde{K}_{k i}: \mathscr{L}_{2 e}^{m_{p_{i}}}[0, \infty) \rightarrow \mathscr{L}_{2 e}^{l_{q_{k}}}[0, \infty) \\
& M=\left[\begin{array}{c}
\tilde{M}_{1} \\
\vdots \\
\tilde{M}_{n_{p}}
\end{array}\right], \tilde{M}_{i} \in \mathbb{C}^{\left(\sum_{k} l_{q_{k}}\right) \times\left(\sum_{k} l_{q_{k}}\right)} \\
& \tilde{M}_{i}=\oplus_{i=1}^{n_{q}}\left(r_{k}^{i} I_{l_{q_{k}}}\right), \text { where } r_{k}^{i}:= \begin{cases}1 & \text { if } \tilde{H}_{i k} \neq 0 \text { or } \tilde{K}_{k i} \neq 0 \\
0 & \text { otherwise }\end{cases}
\end{aligned}
$$

Also define $N_{k}:=\sum_{i=1}^{n_{p}} r_{k}^{i}$ which can be seen as the number of $P_{i}^{\prime} s, Q_{k}$ is interacting with directly. Note that $K\left(\oplus_{i} P_{i}\right) H\left(\oplus_{k} Q_{k}\right)=M^{*}\left(\oplus_{i} G_{i}\right) M$ therefore stability of (3) with $G_{i}$ and $A$ as in (11) implies stability of (7). Hence the approaches described in this section can be used to obtain decentralized conditions for graph separation, which can then lead to corresponding stability conditions. Note that the subsystems $G_{i}$ here involve operators $P_{i}$ and their 'neighboring' $Q_{k}$.

To state the theorem below we consider hermitian valued functions $\Pi^{k}: j \mathbb{R} \rightarrow \mathbb{C}^{2 l_{q_{k}} \times 2 l_{q_{k}}}, k=1, \ldots, n_{q}$, and define $\tilde{\Pi}$ from $\Pi^{k}$ as

$$
\Pi^{k}=\left[\begin{array}{cc}
\Pi_{11}^{k} & \Pi_{12}^{k} \\
\Pi_{12}^{k *} & \Pi_{22}^{k}
\end{array}\right], \quad \tilde{\Pi}^{k}:=\left[\begin{array}{cc}
\oplus_{k} \Pi_{11}^{k} & \oplus_{k} \Pi_{12}^{k} \\
\oplus_{k} \Pi_{12}^{k} * & \oplus_{k} \Pi_{22}^{k}
\end{array}\right]
$$

where $\Pi_{11}^{k}, \Pi_{12}^{k}, \Pi_{22}^{k}$ take values in $\mathbb{C}^{l_{q_{k}} \times l_{q_{k}}}$.

\footnotetext{
${ }^{2}$ The notation used here is that for a matrix of operators $A=\left[\tilde{A}_{i j}\right]$ and a signal $u=\left[u_{1}, \ldots, u_{n}\right]^{T}$ with $u_{j}$ in the domain of $\tilde{A}_{i j}$ then $v=A(u)$ is defined as the signal $v=\left[v_{1}, \ldots, v_{n}\right]^{T}$ where $v_{i}=\sum_{j} \tilde{A}_{i j}\left(u_{j}\right)$. Similarly for a matrix of operators $B=\left[\tilde{B}_{i j}\right], A B$ is the operator defined as $A B(u)=A(B(u))$.
} 
Theorem 7: Consider the interconnection in (3), (11). The graphs of operators $\oplus G_{i}$ and $A$ are $\varepsilon$-quadratically separated if there exists hermitian valued functions $\Pi^{k}$ as in (12) and $\varepsilon>0$ such that

$$
\sigma_{\tilde{\Pi}}(h) \geq \varepsilon\|h\|^{2} \quad \forall h_{i} \in \mathscr{G}_{G_{i}} \quad i=1, \ldots, n
$$

where functional $\sigma_{\tilde{\Pi}}$ is defined as in (2) and $\tilde{\Pi}$ as in (12) with $\Pi_{11}^{k}=0$ and

$$
\left(\Pi_{12}^{k}+\Pi_{12}^{k}+N_{k} \Pi_{22}^{k}\right)(j \omega) \leq 0 \quad \forall \omega \in \mathbb{R}_{+}
$$

Remark 4: Note that (13) is a decentralized condition on each of the $P_{i}$ and its neighboring $Q_{k}$, and (14) is also a decentralized condition on the allowable multipliers.

If now the operators $P_{i}, Q_{k}, H, K$ are linear, conditions by means of the Davis-Wielandt shell can be obtained. These involve additional structure on the allowable multipliers, relative to the conditions in Theorem 7 , they provide, however, a convenient way of verifying graph separation.

Corollary 3: Consider the interconnection in (3), (11) with $G_{i}$ a linear operator with transfer function $\hat{G}_{i} \in$

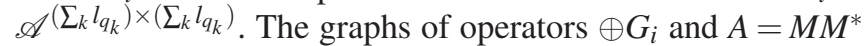
are $\varepsilon$-quadratically separated if $\exists \varepsilon>0$ s.t. for almost all $\omega \in \mathbb{R}_{+}$

$$
\left.C o\left(\cup_{i} D W\left(\hat{G}_{i}(j \omega)\right)\right)\right) \text { and } \overline{D W}\left(M M^{*}\right)
$$

are $\varepsilon$-strictly separated.

Remark 5: Note that $\operatorname{eig}\left(M M^{*}\right) \subseteq\left\{0, N_{k}: k=1, \ldots n_{q}\right\}$, hence $\overline{D W}\left(M M^{*}\right) \subseteq C o\left\{1 / N_{k}, 0, r / N_{k}^{2}: k=1, \ldots n_{q}, r \geq 1\right\}$. The dynamics $Q_{k}$ could also be scaled by $N_{k}$ and $M M^{*}$ accordingly (i.e. $r_{i}^{k}$ is scaled by $1 / N_{k}$ ) such that $\operatorname{eig}\left(M M^{*}\right) \subseteq$ $\{0,1\}$, and therefore $\overline{D W}\left(M M^{*}\right) \subseteq\{1,0, r: r \geq 1\}$. Note also that if $M M^{*}$ is rank deficient then condition (15) needs only to be checked in its first two coordinates.

\section{B. Stability}

Once graph separation has been established stability can then be deduced by employing appropriate homotopy arguments from a stable interconnection as in Theorem 1. It should be noted that for linear operators $G$ and $A$ with transfer functions $\hat{G}, \hat{A}$, graph separation in $\mathscr{L}_{2}$ implies that $I-\hat{G} \hat{A}$ is invertible on the imaginary axis (see Lemma 5 in the appendix). The homotopy employed can then be seen as ensuring that the winding number condition is satisfied in the Nyquist stability criterion [26].

A convenient way to establish this homotopy is to assume that operators $G$ and $A$ are bounded and then consider a continuous perturbation from open loop by means of a scalar as in [11]. In fact, if graph separation is established by a functional $\sigma_{\Pi}$ with $\Pi_{11}(j \omega) \leq 0, \Pi_{22}(j \omega) \geq 0$ then graph separation is also implied throughout this homotopy from open-loop, and therefore stability of the system is also implied. This is stated in the Theorem below.

Theorem 8: Consider the interconnection in (3) with $G_{i}$ and $A$ as defined in Theorems 3, 4, 5, 7 respectively. Then the conditions for graph separation in the theorems are also sufficient for stability of the corresponding interconnections if

(i) $G_{i}$ and $A$ are bounded operators. (ii) $\Pi_{11}^{i}(j \omega) \leq 0, \quad \Pi_{11}(j \omega) \leq 0$ and $\Pi_{22}^{i}(j \omega) \geq 0$, $\Pi_{22}(j \omega) \geq 0, \Pi_{22}^{k}(j \omega) \geq 0$.

(iii) The interconnection between $G$ and $\tau A$ is well posed for all $\tau \in[0,1]$.

Remark 6: Note that the condition $\Pi_{22}(j \omega) \geq 0$ can be relaxed if there are dynamics $G_{i}^{0}$ for which the interconnection is stable, and satisfy the corresponding graph separation condition. Stability then follows by considering the homotopy $G_{\tau}=\tau G_{i}+(1-\tau) G, A_{\tau}=A, \tau \in[0,1]$.

Note also that analogous continuity arguments are satisfied in Corollaries 2, 3.

Corollary 4: Consider the interconnection in (3) with $G_{i}$ and $A$ as in Corollary 2. The graph separation condition in Corollary 2 is also sufficient for stability of the interconnection.

Corollary 5: Consider the interconnection in (3) with $G_{i}$ and $A$ as in Corollary 3 with $n_{q}>1$. The graph separation condition in the Corollary 3 is also sufficient for stability of the interconnection.

It should be noted that the different stability conditions have different relative merits. For example the conditions in Theorem 4 and Corollary 3 when the interconnection matrix is skew symmetric recover passivity type results where stability is guaranteed for an arbitrary network (without any need for scaling) when the individual subsystems $P_{i}, Q_{k}$ are strictly passive. This is not necessarily the case in Theorem 7, due to the rank deficiency of $G_{i}$ and $M M^{*}$, nevertheless the way neighboring dynamics are taken into account can often reduce conservatism. Other classes of multipliers and network transformations could also be possible (e.g. [27]).

It should also be noted that the results in Theorem 7 (and Corollary 3) include many of the features exploited in [5], while providing various extensions. In particular one of the main ideas in [5] is to use a similarity transformation in the return ratio (this is a special case of the multipliers in Theorem 7), and then get a spectral bound on the return ratio using numerical ranges that are overestimated by means of S-hulls. The definition of the S-hull is closely related to that of the numerical range with a main difference being that the scaling is done by means of the 1-norm rather than the 2-norm. More precisely, consider the interconnection in (11), with $P_{i}, Q_{i}$ being linear operators with transfer functions $\hat{P}_{i} \in \mathscr{A}, \hat{Q}_{i} \in \mathscr{A}$. Also let $H=K^{*}$ be a $0-1$ matrix. Then a stability condition from [5] is

$$
1 \notin \bigcup_{\omega \in \overline{\mathbb{R}}_{+}} \rho\left(|H||H|^{T}\right) \operatorname{Co}\left\{\cup_{i} S\left\{\hat{P}_{i}(j \omega) \hat{Q}_{k}(j \omega): H_{i k} \neq 0\right\}\right\}
$$

where the S-hull S(.) is given here by (Lemma 2.2.1 in [28])

$$
\begin{aligned}
& S\left\{\hat{P}_{i}(j \omega) \hat{Q}_{k}(j \omega): H_{i k} \neq 0\right\} \\
& \quad=\left\{v^{*} G_{i}(j \omega) v: v \in \mathbb{C}^{n_{q}},\|v\|_{1} \leq 1\right\}=: \mathscr{S}\left(G_{i}(j \omega)\right)
\end{aligned}
$$

with $G_{i}=\left(\oplus_{k} \sqrt{\hat{Q}_{k}}\right)\left(H_{i}^{r}\right)^{*} \hat{P}_{i} H_{i}^{r}\left(\oplus_{k} \sqrt{\hat{Q}_{k}}\right)$. It can be shown that a special case of the multipliers in Theorem 7 (Corollary 3 with a similarity transformation in the return ratio) leads 
to the stability condition

$$
\left[\frac{1}{\max _{k} N_{k}}, \frac{1}{\min _{k} N_{k}}\right] \cap \bigcup_{\omega \in \overline{\mathbb{R}}_{+}} \operatorname{Co}\left(\cup_{i} W\left\{G_{i}(j \omega)\right\}\right)=\emptyset
$$

where $N_{k}:=\sum_{i} H_{i k}$ and $W($.$) is the projection of the Davis$ Wielandt shell in its first two coordinates. This condition is implied if

$$
1 \notin \bigcup_{\omega \in \overline{\mathbb{R}}_{+}}\left(\max _{k} N_{k}\right) \operatorname{Co}\left(\cup_{i} W\left\{G_{i}(j \omega) \cup 0\right\}\right)
$$

and note that ${ }^{3}$

$$
\frac{1}{N_{i}} W\left\{G_{i} \cup 0\right\} \subseteq \mathscr{S}\left(G_{i}\right) \subseteq W\left\{G_{i} \cup 0\right\}
$$

where $N_{i}=\sum_{k} H_{i k}$. In order to transform $H$ to a scaled version $\bar{H}$ such that $\sum_{i} \bar{H}_{i k} \leq 1$, each of the $Q_{k}$ will need to be scaled by $N_{k}$, whereas to ensure that $\rho\left(|\bar{H}||\bar{H}|^{T}\right) \leq 1$ in a decentralized way, $Q_{k}$ will need to be scaled be $N_{k}$ but also $P_{i}$ by $N_{i}$. This therefore counterbalances the fact that the S-hull is included in the corresponding numerical range (see (19)) and hence (18) becomes less restrictive than (16). Analogous arguments also hold when $\hat{H}$ is complex valued.

Finally, applications for the stability conditions considered in the paper include Internet congestion and consensus protocols, in analogy with those considered in [5], [28] with the conditions being also relevant for related problems such as distributed control in power distribution networks and higher order consensus protocols.

\section{CONCLUSIONS}

It was shown in the paper how different forms of separating functionals can be used to derive decentralized stability certificates for large scale networks. These are based on an exploitation of the interconnection structure, which can lead to extended classes of such functionals with reduced conservatism. Interpretations of the stability certificates by means of disjoint convex shells have also been discussed.

\section{APPENDIX}

Lemma 5: Consider the interconnection in (1) where $G$ and $\Delta$ are linear operators with transfer function in $\hat{G} \in$ $\mathscr{A}^{n \times m}, \hat{\Delta} \in \mathscr{A}^{m \times n}$. If the graphs of $G$ and $\Delta$ are $\varepsilon$ quadratically separated, then $I-\hat{G}(j \omega) \hat{\Delta}(j \omega)$ is invertible for all $\omega \in \overline{\mathbb{R}}$.

\section{REFERENCES}

[1] P. Moylan and D. Hill, "Stability criteria for large-scale systems," IEEE Transactions on Automatic Control, vol. 23, no. 2, pp. 143-149, 1978.

[2] M. Vidyasagar, Input-Output Analysis of Large-Scale Interconnected Systems. Springer-Verlag, 1981.

[3] J. Fax and R. Murray, "Information flow and cooperative control of vehicle formations," IEEE Transactions on Automatic Control, vol. 49, no. 9, pp. 1465-1476, 2004.

[4] I. Lestas and G. Vinnicombe, "Scalable robustness for consensus protocols with heterogeneous dynamics," in Proceedings of IFAC world congress, July 2005.

${ }^{3}$ Follows from the fact that for $x \in \mathbb{C}^{n} \frac{1}{\sqrt{n}}\|x\|_{1} \leq\|x\|_{2} \leq\|x\|_{1}$.
[5] _ - "Scalable decentralized robust stability certificates for networks of interconnected heterogeneous dynamical systems," IEEE Transactions on Automatic Control, vol. 51, no. 10, pp. 1613-1625, October 2006.

[6] U. Jonsson, C.-Y. Kao, and H. Fujioka, "A popov criterion for networked systems," Systems and Control Letters, vol. 56, pp. 603610, 2007.

[7] C.-Y. Kao, U. Jonsson, and H. Fujioka, "Characterization of robust stablity of a class of interconnected systems," Automatica, vol. 45, pp. 217-224, 2009.

[8] U. Jonsson and C.-Y. Kao, "A scalable robust stability criterion for systems with heterogeneous LTI components," IEEE Transactions on Automatic Control, vol. 55, no. 10, pp. 2219-2234, 2010.

[9] M. Arcak, "Passivity as a design tool for group coordination," IEEE Transactions on Automatic Control, vol. 52, no. 8, pp. 1380-1390, August 2007.

[10] R. Srikant, The mathematics of Internet congestion control. Birkhauser, 2004.

[11] A. Megretski and A. Rantzer, "System analysis via integral quadratic constraints," IEEE Transactions on Automatic Control, vol. 42, no. 6 , pp. 819-830, 1997.

[12] I. Lestas, "Scalable decentralized control and the Davis-Wieland shell," in Proceedings of Mathematical Theory of Networks and Systems, July 2010. An extended version is under review for journal publication.

[13] _ , "On network stability, graph separation, interconnection structure and convex shells," Cambridge University, Engineering Department, Tech. Rep., January 2011, CUED/F-INFENG/TR.662.

[14] R. T. Rockafellar, Convex Analysis. Princeton, N.J.: Princeton University Press, 1970.

[15] F. M. Callier and C. A. Desoer, "An algebra of transfer functions for distributed linear time-invariant systems," IEEE Transactions on Circuits and Systems, vol. 25, no. 9, pp. 651-662, 1978.

[16] C. A. Desoeer and M. Vidyasagar, Feedback systems: input-output properties. New York: Academic Press, 1975.

[17] F. M. Callier and C. A. Desoer, "Simplifications and clarifications on the paper "an algebra of transfer functions for distributed linear time-invariant systems"," IEEE Transactions on Circuits and Systems, vol. 27, no. 4, pp. 320-323, 1980.

[18] A. Rantzer and A. Megretski, "Stability criteria based on integral quadratic constraints," in 35th Conference on Decision and Control, 1996.

[19] T. T. Georgiou and M. C. Smith, "Robustness analysis of nonlinear feedback systems: An input-output approach," IEEE Transactions on Automatic Control, vol. 42, no. 9, pp. 1200-1221, 1997.

[20] V. A. Yakubovich, "Frequncy conditions for the absolute stability of control systems with several nonlinear or linear nonstationary units," Automatica and telemechanica, vol. 28, no. 6, pp. 5-30, 1967.

[21] C. Davis, "The shell of a Hilbert space operator," Acta Sci. Math, vol. 29, pp. 69-86, 1968.

[22] H. Wielandt, "Inclusion theorems for eigenvalues," US. Department of Commerce, National Bureau of Standards, Applied Mathematics Series, vol. 29, pp. 75-78, 1953.

[23] R. A. Horn and C. R. Johnson, Topics in Matrix Analysis. Cambridge University Press, 1991.

[24] P. Binding, "Hermitian forms and the fibration of spheres," Proceedings of the American Mathematical Society, vol. 94, no. 4, pp. 581584, 1985.

[25] U. Jonsson, C.-Y.Kao, and H. Fujioka, "Low dimensional stability criteria for large-scale interconnected systems," in Proceedings of European Control Conference, 2007.

[26] C. A. Desoer and Y. T. Yang, "On the generalized Nyquist stability criterion," IEEE Transactions on Automatic Control, vol. 25, pp. 187196, 1980.

[27] U. Jonsson, "Primal and dual criteria for robust stability and their application to systems interconnected over a bipartite graph," in Proceedings of American Control Conference, 2010.

[28] I. Lestas, "On the analysis of scalable heterogeneous networks," Ph.D. dissertation, University of Cambridge, 2006. 